\title{
Seismo Mechanical Energy Fractal Dimension for Characterizing Shajara Reservoirs of the Permo-Carboniferous Shajara Formation, Saudi Arabia
}

\author{
Khalid Elyas Mohamed Elameen Alkhidir* \\ Department of Petroleum and Natural Gas Engineering, College of Engineering, King Saud University, Riyadh, \\ Saudi Arabia.
}

*Corresponding Author: Khalid Elyas Mohamed Elameen Alkhidir, Department of Petroleum and Natural Gas Engineering, College of Engineering, King Saud University, Riyadh, Saudi Arabia.

\begin{abstract}
The quality of a reservoir can be described in details by the application of seismo mechanical energy fractal dimension. The objective of this research is to calculate fractal dimension from the relationship among seismo mechanical energy, maximum seismo mechanical energy and wetting phase saturation and to confirm it by the fractal dimension derived from the relationship among capillary pressure and wetting phase saturation. In this research, porosity was measured on real collected sandstone samples and permeability was calculated theoretically from capillary pressure profile measured by mercury intrusion techniques. Two equations for calculating the fractal dimensions have been employed. The first one describes the functional relationship between wetting phase saturation, seismo mechanical energy, maximum seismo mechanical energy and fractal dimension. The second equation implies to the wetting phase saturation as a function of capillary pressure and the fractal dimension. Two procedures for obtaining the fractal dimension have been developed. The first procedure was done by plotting the logarithm of the ratio between seismo mechanical energy and maximum seismo mechanical energy versus logarithm wetting phase saturation. The slope of the first procedure $=3-$ Df (fractal dimension). The second procedure for obtaining the fractal dimension was completed by plotting the logarithm of capillary pressure versus the logarithm of wetting phase saturation. The slope of the second procedure = Df -3. On the basis of the obtained results of the constructed stratigraphic column and the acquired values of the fractal dimension, the sandstones of the Shajara reservoirs of the Shajara Formation were divided here into three units. The gained units from bottom to top are: Lower Shajara Seismo Mechanical Energy Fractal Dimension Unit, Middle Shajara Seismo Mechanical Energy Fractal Dimension Unit, and Upper Shajara Seismo Mechanical Energy Fractal Dimension Unit. The fractal dimension was found to increase with increasing permeability and grain size.
\end{abstract}

Keywords: Shajara reservoirs, Shajara Formation, Seismo mechanical energy fractal dimension, Capillary pressure fractal dimension

\section{INTRODUCTION}

Seismo electric effects related to electro kinetic potential, dielectric permitivity, pressure gradient, fluid viscosity, and electric conductivty was first reported by [1]. Capillary pressure follows the scaling law at low wetting phase saturation was reported by [2]. Seismo electric phenomenon by considering electro kinetic coupling coefficient as a function of effective charge density, permeability, fluid viscosity and electric conductivity was reported by [3]. The magnitude of seismo electric current depends on porosity, pore size, zeta potential of the pore surfaces, and elastic properties of the matrix was investigated by [4]. The tangent of the ratio of converted electic field to pressure is approximately in inverse proportion to permeability was studied by [5]. Permeability inversion from seismoelectric $\log$ at low frequency was studied by [6]. They reported that, the tangent of the ratio among electric excitation intensity and pressure field is a function of porosity, fluid viscosity, frequency, tortuosity and fluid density and Dracy permeability. A decrease of seismo electric frequencies with increasing water content was reported by [7]. An increase of seismo electric transfer function with increasing water saturation was studied by [8]. An increase of dynamic seismo electric transfer function with decreasing fluid conductivity was described by [9]. The amplitude of seismo electric signal increases 
with increasing permeability which means that the seismo electric effects are directly related to the permeability and can be used to study the permeability of the reservoir was illustrated by [10]. Seismo electric coupling is frequency dependent and decreases expontialy when frequency increases was demonstrated by [11]. An increase of permeability with increasing seismo magnetic moment and seismo diffusion coefficiernt fractal dimension was reported by [12, 13]. An increase of, molar enthalpy, work, electro kinetic, bubble pressure and pressure head fractal dimensions with permeability increasing and grain size was described by $[14,15,16,17]$.

\section{MATERIAL AND MethodS}

Porosity was measured on collected sandstone samples and permeability was calculated from the measured capillary pressure by mercury intrusion techniques. Two procedures for obtaining the fractal dimension have been developed. The first procedure was done by plotting the logarithm of the ratio between seismo mechanical energy and maximum seismo mechanical energy versus logarithm wetting phase saturation. The slope of the first procedure $=3$ - Df (fractal dimension). The second procedure for obtaining the fractal dimension was completed by plotting the logarithm of capillary pressure versus the logarithm of wetting phase saturation. The slope of the second procedure $=$ Df -3 .

The seismo mechanical energy can be scaled as

$S w=\left[\frac{\operatorname{SME}^{\frac{1}{4}}}{\operatorname{SME}_{\max }^{\frac{1}{4}}}\right]^{[3-D f]}$

Where $\mathrm{Sw}$ the water saturation, SME seismo mechanical energy in Joule, $\mathrm{SME}_{\max }$ the maximum seismo mechanical energy in Joule, and Df the fractal dimension.

Equation 1 can be proofed from

$\mathbf{V}=$

$\left[\frac{\mathrm{k} * \boldsymbol{\rho f} * \boldsymbol{\omega}^{2} * \mathrm{Us}}{\mu}\right]$

Where $\mathrm{V}$ the velocity in meter / second, $\mathrm{k}$ the permeability in square meter, $\rho$ f the fluid density in kilo gram /cubic meter, $\omega$ the seismic angular frequency in hertz, Us the seismic displacement in meter, and $\mu$ the fluid viscosity in pascal * second.

The density can be scaled as

$$
\boldsymbol{\rho} \mathbf{f}=\left[\frac{\mathbf{m}}{\mathbf{v}}\right]
$$

Where $\rho$ f the fluid density in kilo gram / cubic meter, $m$ the mass in kio gram, and $v$ the volume in cubic meter.

Insert equation 3 into equation 2

$\mathbf{V}=\left[\frac{\mathbf{k} * \mathbf{m} * \boldsymbol{\omega}^{2} * \mathbf{U s}}{\mathbf{v} * \boldsymbol{\mu}}\right]$

The mass $\mathrm{m}$ can be scaled as

$\mathbf{m}=\left[\begin{array}{l}\mathbf{F} \\ \mathbf{g}\end{array}\right]$

Where $\mathrm{m}$ the mass in kilo gram, $\mathrm{F}$ the seismo mechanical force in newton, and $\mathrm{g}$ the gravitational acceleration in meter / square second

Insert equation 5 into equation 4

$\mathbf{V}=\left[\frac{\mathbf{k} * \mathbf{F} * \mathbf{\omega}^{2} * \mathbf{U s}}{\mathbf{g} * \mathbf{V} * \boldsymbol{\mu}}\right]$ 
The seismo mechanical force $\mathrm{F}$ can be scaled as

$\mathbf{F}=\left[\frac{\mathbf{S M E}}{\mathbf{d}}\right]$

Where $\mathrm{F}$ the seismo mechanical force in newton, SME the seismo mechanical energy in Joule, and d the distance in meter.

Insert equation 7 into equation 6

$\mathbf{V}=\left[\frac{\mathbf{k} * \mathbf{S M E} * \boldsymbol{\omega}^{2} * \mathbf{U s}}{\mathbf{d} * \mathbf{g} * \mathbf{V} * \boldsymbol{\mu}}\right]$

The velocity $\mathrm{V}$ can be scaled as

$\mathbf{V}=\left[\frac{\mathbf{Q}}{\mathbf{A}}\right]$

Where V the velocity in meter / second, Q the flow rate in cubic meter / second, and A the area in square meter.

Insert equation 9 into equation 8

$\left[\frac{\mathbf{Q}}{\mathbf{A}}\right]=\left[\frac{\mathbf{k} * \mathbf{S M E} * \boldsymbol{\omega}^{2} * \mathbf{U s}}{\mathbf{d} * \mathbf{g} * \mathbf{V} * \boldsymbol{\mu}}\right]$

The flow rate can be scaled as

$\mathbf{Q}=\left[\frac{3.14 * \mathbf{r}^{4} * \Delta \mathbf{p}}{\mathbf{8} * \boldsymbol{\mu} * \mathbf{L}}\right]$

Where $\mathrm{Q}$ the flow rate in cubic meter / second, $\mathrm{r}$ the pore radius in meter, $\Delta \mathrm{p}$ the differential pressure in pascal, $\mu$ the fluid viscosity in pascal $*$ second, and $L$ the length in meter.

Insert equation 11 into equation 10

$\left[\frac{3.14 * \mathbf{r}^{4} * \Delta \mathbf{p}}{\mathbf{A} * \mathbf{8} * \boldsymbol{\mu} * \mathbf{L}}\right]=\left[\frac{\mathbf{k} * \mathbf{S M E} * \boldsymbol{\omega}^{2} * \mathbf{U s}}{\mathbf{d} * \mathbf{g} * \mathbf{v} * \boldsymbol{\mu}}\right]$

Equation 12 after pore radius rearrangement will become

$\mathbf{r}^{4}=\left[\frac{\mathbf{A} * \mathbf{8} * \boldsymbol{\mu} * \mathbf{L} * \mathbf{k} * \mathbf{S M E} * \boldsymbol{\omega}^{2} * \mathbf{U s}}{\mathbf{3 . 1 4} * \Delta \mathbf{p} * \mathbf{d} * \mathbf{g} * \mathbf{v} * \boldsymbol{\mu}}\right]$

The maximum pore radius can be scaled as

$\mathbf{r}_{\text {max }}^{\mathbf{4}}=\left[\frac{\mathbf{A} * \mathbf{8} * \boldsymbol{\mu} * \mathbf{L} * \mathbf{k} * \mathbf{S M E}_{\max } * \boldsymbol{\omega}^{2} * \mathbf{U s}}{\mathbf{3 . 1 4} * \Delta \mathbf{p} * \mathbf{d} * \mathbf{g} * \mathbf{v} * \boldsymbol{\mu}}\right]$

Divide equation 13 by equation 14

$\left[\frac{\mathbf{r}^{4}}{\mathbf{r}_{\max }^{4}}\right]=\left[\frac{\left[\frac{\mathbf{A} * \mathbf{8} * \boldsymbol{\mu} * \mathbf{L} * \mathbf{k} * \mathbf{S M E} * \boldsymbol{\omega}^{2} * \mathbf{U s}}{3.14 * \Delta \mathbf{p} * \mathbf{d} * \mathbf{g} * \mathbf{V} * \boldsymbol{\mu}}\right]}{\left[\frac{\mathbf{A} * \mathbf{8} * \boldsymbol{\mu} * \mathbf{L} * \mathbf{k} * \mathbf{S M E} \mathbf{E}_{\max } * \boldsymbol{\omega}^{2} * \mathbf{U s}}{\mathbf{3 . 1 4} * \Delta \mathbf{p} * \mathbf{d} * \mathbf{g} * \mathbf{V} * \boldsymbol{\mu}}\right]}\right]$

Equation 15 after simplification will become

$\left[\frac{\mathbf{r}^{4}}{\mathbf{r}_{\max }^{4}}\right]=\left[\frac{\mathrm{SME}}{\mathrm{SME}_{\max }}\right]$

Take the forth root of equation 16

$\sqrt[4]{\left[\frac{\mathbf{r}^{4}}{\mathbf{r}_{\text {max }}^{4}}\right]}=\sqrt[4]{\left[\frac{S M E}{S M E_{\text {max }}}\right]}$

International Journal of Petroleum and Petrochemical Engineering (IJPPE) 
Equation 17 after simplification will become

$\left[\frac{r}{r_{\text {max }}}\right]=\left[\frac{S M^{\frac{1}{4}}}{S M_{\text {max }}^{\frac{1}{4}}}\right]$

Take the logarithm of equation 18

$\log \left[\frac{r}{r_{\text {max }}}\right]=\log \left[\frac{S M^{\frac{1}{4}}}{S M E_{\text {max }}^{\frac{1}{4}}}\right]$

But, $\log \left[\frac{r}{r_{\text {max }}}\right]=\left[\frac{\log S w}{3-D f}\right]$

Insert equation 20 into equation 19

$$
\left[\frac{\log S w}{3-D f}\right]=\log \left[\frac{S M E^{\frac{1}{4}}}{S M E_{\text {max }}^{\frac{1}{4}}}\right]
$$

Equation 21 after log removal will become

$S w=\left[\frac{S E^{1 / 4}}{S M E_{\text {max }}^{\frac{1}{4}}}\right]^{[3-D f]}$

The capillary pressure can be scaled as

$\log \mathbf{S w}=[\mathrm{Df}-3] * \mathbf{P c}+$ constant

Where $\mathrm{Sw}$ the water saturation, Pc the capillary pressure and Df the fractal dimension

\section{RESULTS AND DISCUSSION}

Based on field observation the Shajara Reservoirs of the Permo-Carboniferous Shajara Formation were divided here into three units as described in Figure1.These units from bottom to top are: Lower Shajara Reservoir, Middle Shajara reservoir, and Upper Shajara Reservoir. Their acquired results of the seismo mechanical energy fractal dimension and capillary pressure fractal dimension are displayed in Table 1. Based on the attained results it was found that the seismo mechanical energy fractal dimension is equal to the capillary pressure fractal dimension. The maximum value of the fractal dimension was found to be 2.7872 assigned to sample SJ13 from the Upper Shajara Reservoir as verified in Table 1. Whereas the minimum value of the fractal dimension 2.4379 was reported from sample SJ3 from the Lower Shajara reservoir as displayed in Table1. The seismo mechanical energy fractal dimension and capillary pressure fractal dimension were observed to increase with increasing permeability as proofed in Table1 owing to the possibility of having interconnected channels.

The Lower Shajara reservoir was denoted by six sandstone samples (Figure 1), four of which label as SJ1, SJ2, SJ3 and SJ4 were carefully chosen for capillary pressure measurement as established in Table1. Their positive slopes of the first procedure $\log$ of the ratio of seismo mechanical energy (SME) to maximum seismo mechanical energy $\left(\mathrm{SME}_{\max }\right)$ versus log wetting phase saturation $(\mathrm{Sw})$ and negative slopes of the second procedure log capillary pressure $(\mathrm{Pc})$ versus log wetting phase saturation (Sw) are explained in Figure 2, Figure 3, Figure 4, Figure 5 and Table 1. Their seismo mechanical energy fractal dimension and capillary pressure fractal dimension values are revealed in Table 1. As we proceed from sample $\mathrm{SJ} 2$ to $\mathrm{SJ} 3$ a pronounced reduction in permeability due to compaction was described from $1955 \mathrm{md}$ to $56 \mathrm{md}$ which reflects decrease in seismo mechanical energy fractal dimension from 2.7748 to 2.4379 as quantified in table 1. Again, an increase in grain 
Seismo Mechanical Energy Fractal Dimension for Characterizing Shajara Reservoirs of the PermoCarboniferous Shajara Formation, Saudi Arabia

size and permeability was proved from sample SJ4 whose seismo mechanical energy fractal dimension and capillary pressure fractal dimension was found to be 2.6843 as pronounced in Table 1 .

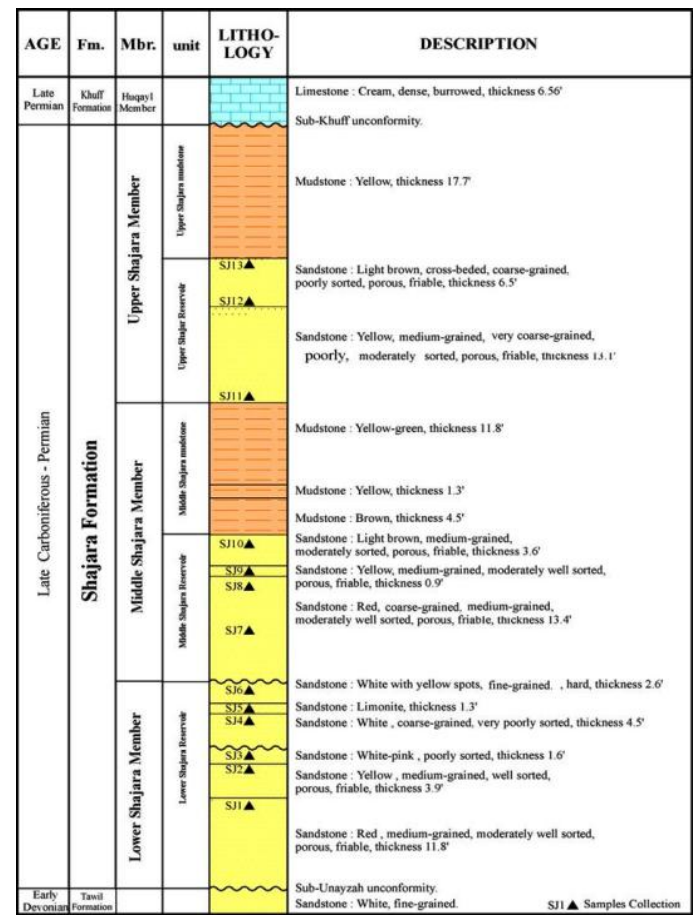

Figure1. Surface type section of the Shajara reservoirs of the Permo-Carboniferous Shajara Formation at latitude $26^{\circ} 52^{\prime} 17.4^{\prime \prime}$ longitude $43^{\circ} 36^{\prime} 18^{\prime \prime}$

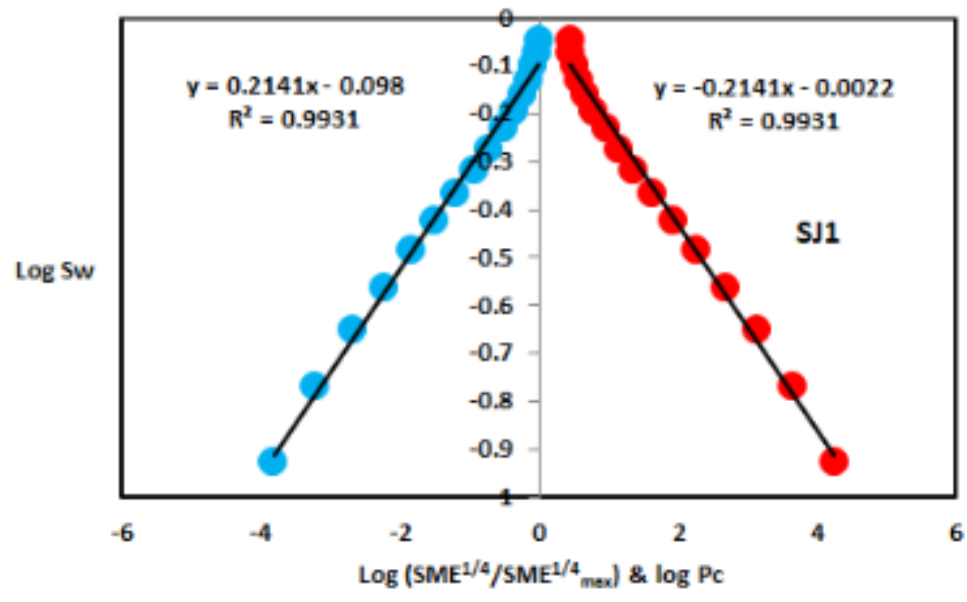

Figure2. $\log \left(S M E^{1 / 4} / S M E_{\max }^{1 / 4}\right) \& \log$ pc versus $\log S w$ for sample SJ1

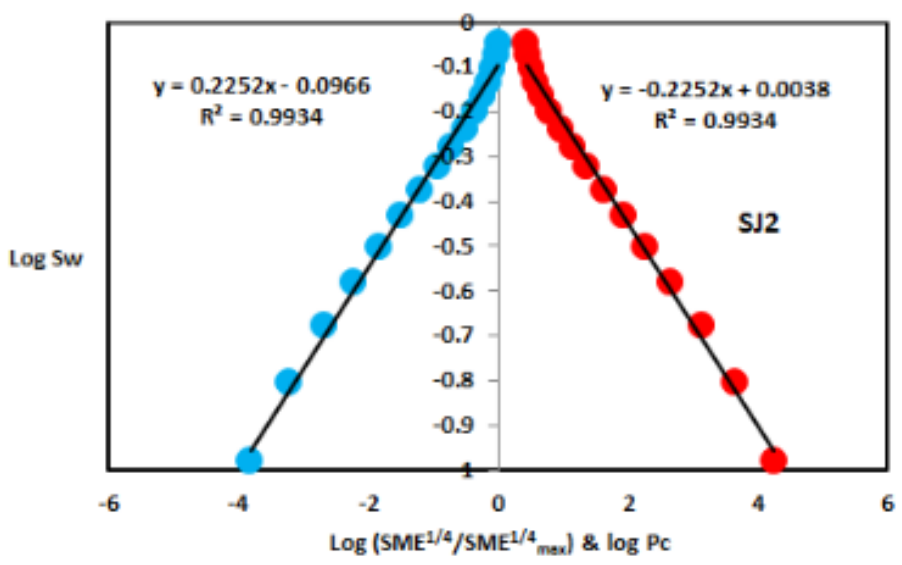

Figure 3. $\log \left(S M E^{1 / 4} / S M E_{\max }^{1 / 4}\right)$ \& $\log$ pc versus log $S w$ for sample $S J 2$ 
Seismo Mechanical Energy Fractal Dimension for Characterizing Shajara Reservoirs of the PermoCarboniferous Shajara Formation, Saudi Arabia

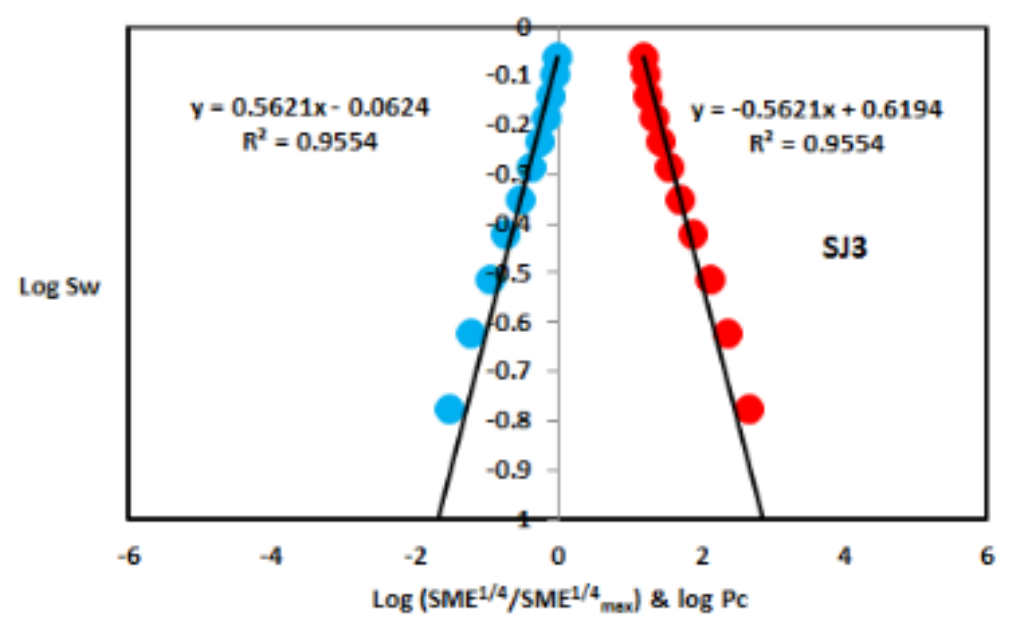

Figure4. $\log \left(S M E^{1 / 4} / S M E_{\max }^{1 / 4}\right) \& \log$ pc versus $\log S w$ for sample SJ3

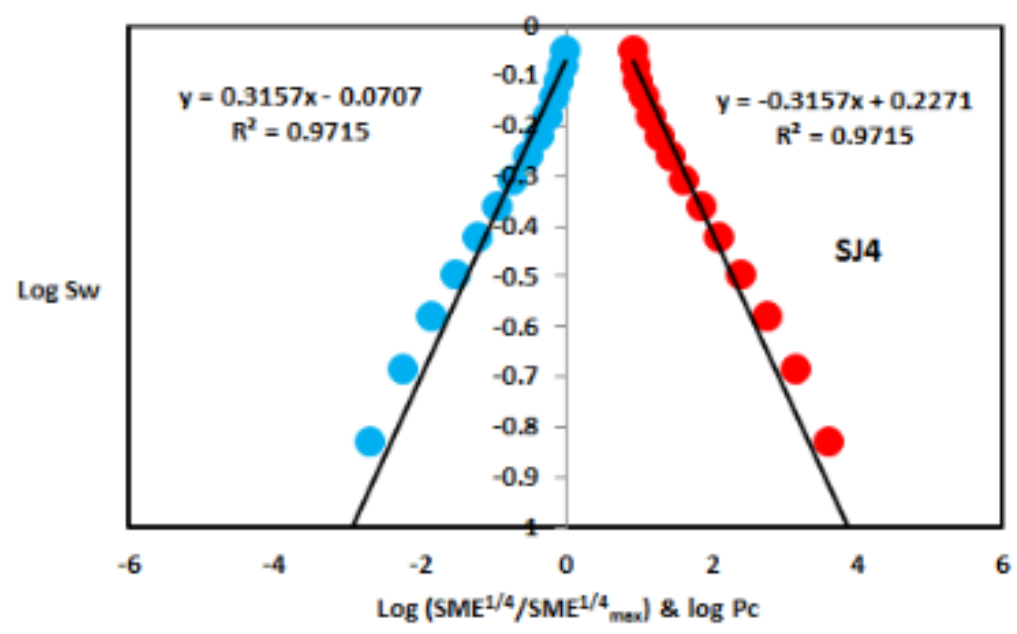

Figure5. $\log \left(S M E^{1 / 4} / S M E_{\max }^{1 / 4}\right) \& \log$ pc versus $\log$ Sw for sample SJ4

In contrast, the Middle Shajara reservoir which is separated from the Lower Shajara reservoir by an unconformity surface as shown in Figure 1. It was nominated by four samples (Figure 1), three of which named as SJ7, SJ8, and SJ9 as clarified in Table1 were chosen for capillary pressure measurements as described in Table 1. Their positive slopes of the first procedure and negative slopes of the second procedure are shown in Figure 6, Figure 7 and Figure 8 and Table 1. Furthermore, their seismo mechanical energy fractal dimensions and capillary pressure fractal dimensions show similarities as defined in Table 1.Their fractal dimensions are higher than those of samples SJ3 and SJ4 from the Lower Shajara Reservoir due to an increase in their permeability as explained in table 1.

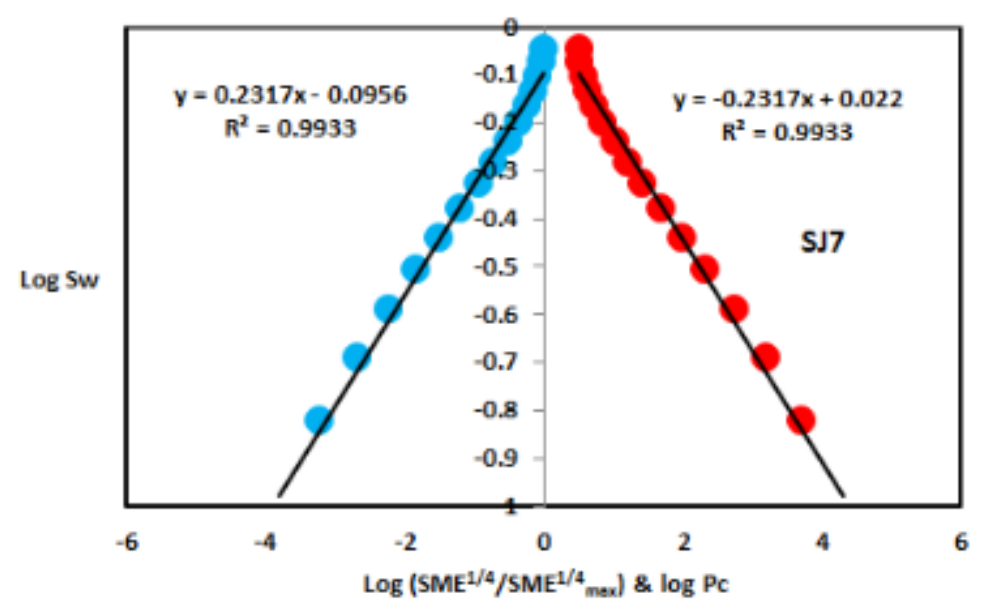

Figure6. $\log \left(S M E^{1 / 4} / S M E_{\max }^{1 / 4}\right) \& \log$ pc versus $\log$ Sw for sample SJ7 
Seismo Mechanical Energy Fractal Dimension for Characterizing Shajara Reservoirs of the PermoCarboniferous Shajara Formation, Saudi Arabia

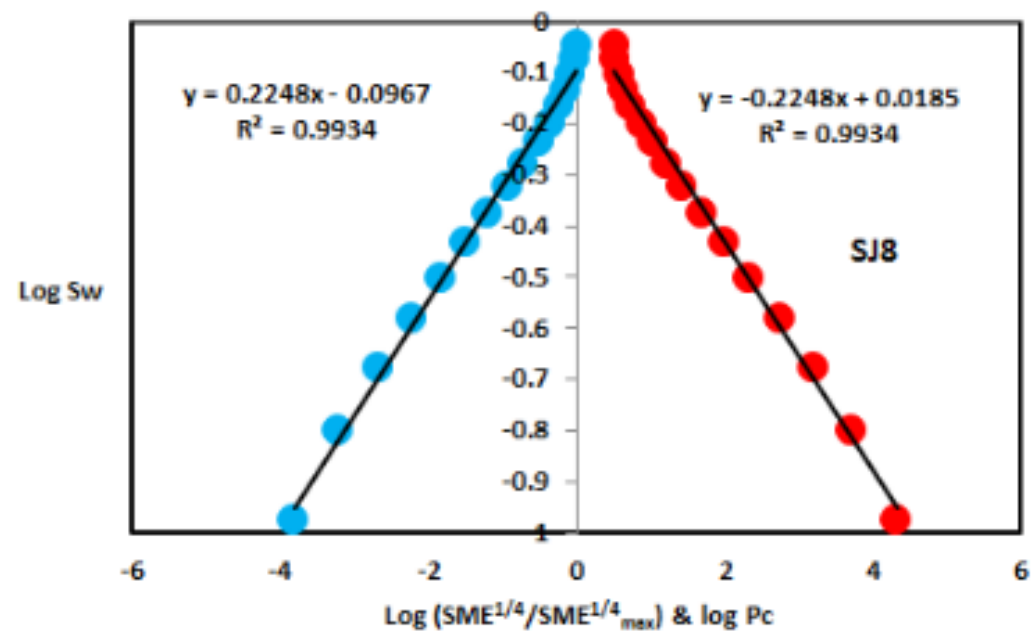

Figure7. $\log \left(S M E^{1 / 4} / S M E_{\max }^{1 / 4}\right) \& \log$ pc versus $\log$ Sw for sample SJ8

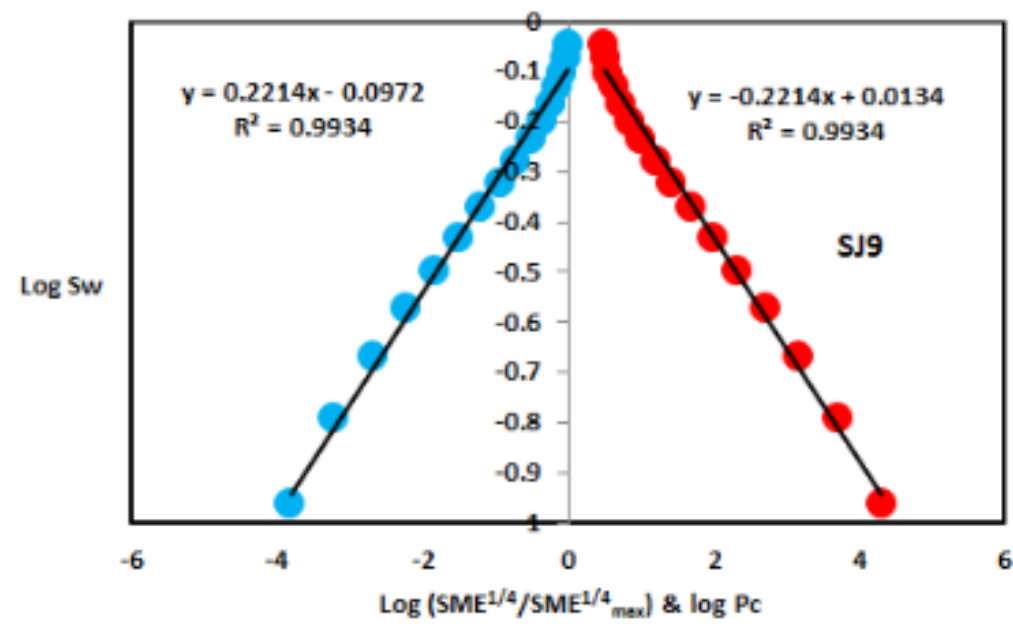

Figure8. $\log \left(S M E^{1 / 4} / S M E_{\max }^{1 / 4}\right) \& \log$ pc versus $\log S w$ for sample SJ9

On the other hand, the Upper Shajara reservoir was separated from the Middle Shajara reservoir by yellow green mudstone as shown in Figure 1. It is defined by three samples so called SJ11, SJ12, SJ13 as explained in Table 1. Their positive slopes of the first procedure and negative slopes of the second procedure are displayed in Figure 9, Figure 10 and Figure 11 and Table 1. Moreover, their seismo mechanical energy fractal dimension and capillary pressure fractal dimension are also higher than those of sample SJ3 and SJ4 from the Lower Shajara Reservoir due to an increase in their permeability as simplified in table 1 .

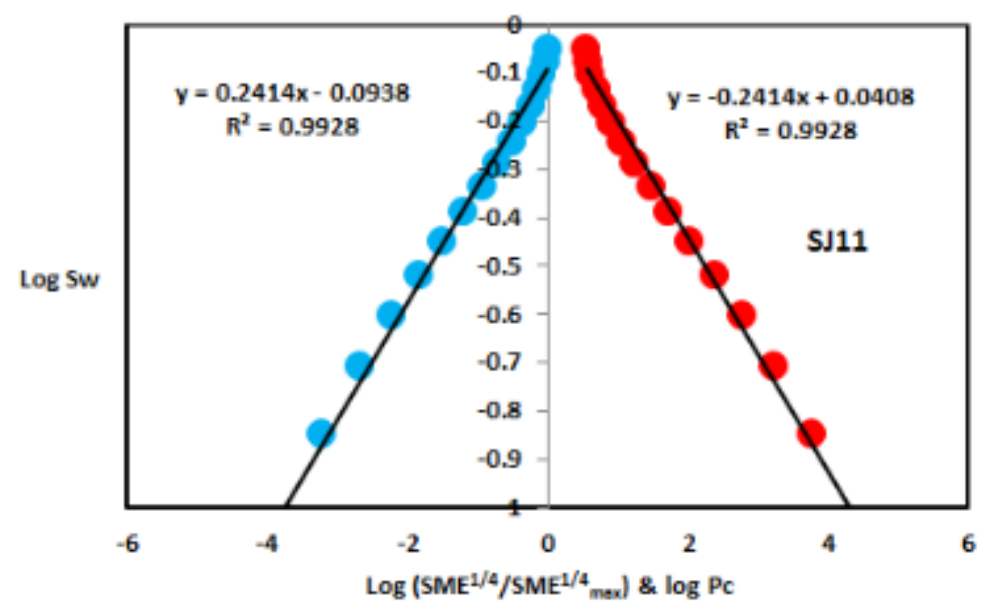

Figure9. $\log \left(S M E^{1 / 4} / S M E_{\max }^{1 / 4}\right) \& \log p c$ versus log Sw for sample SJ11 
Seismo Mechanical Energy Fractal Dimension for Characterizing Shajara Reservoirs of the PermoCarboniferous Shajara Formation, Saudi Arabia

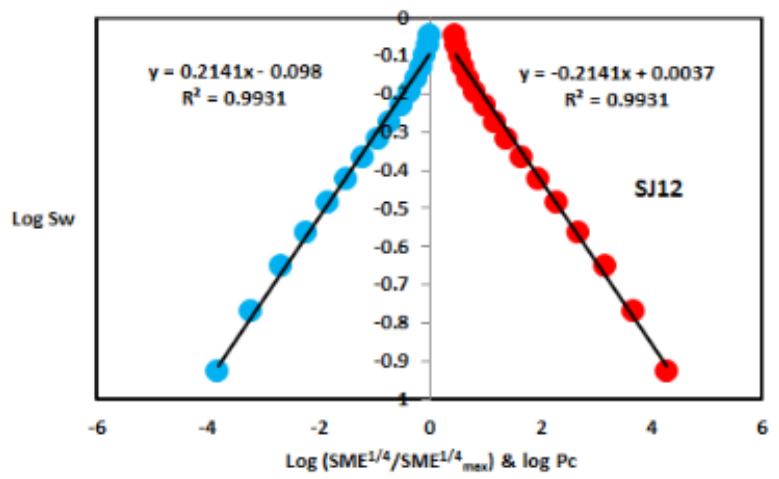

Figure10. $\log \left(S M E^{1 / 4} / S M E_{\max }^{1 / 4}\right)$ \& $\log p c$ versus log $S w$ for sample $S J 12$

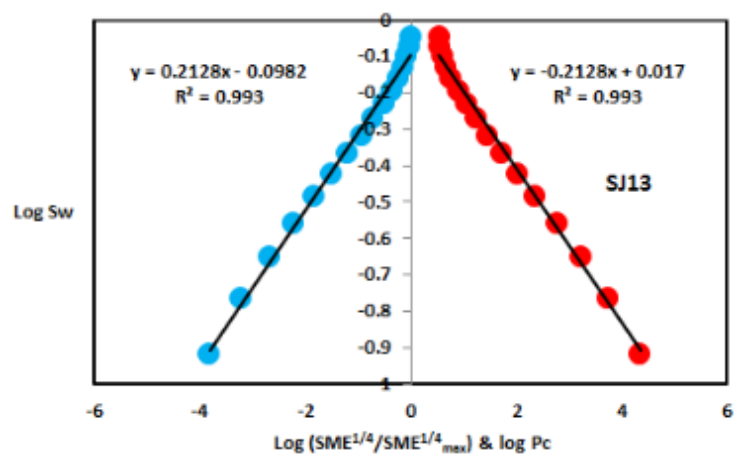

Figure11. $\log \left(S M E^{1 / 4} / S M E_{\max }^{1 / 4}\right)$ \& $\log p c$ versus $\log S w$ for sample $S J 13$

Overall a plot of positive slope of the first procedure versus negative slope of the second procedure as described in Figure 12 reveals three permeable zones of varying Petrophysical properties. These reservoir zone were also confirmed by plotting seismo mechanical energy fractal dimension versus capillary pressure fractal dimension as described in Figure 13. Such variation in fractal dimension can account for heterogeneity which is a key parameter in reservoir quality assessment.

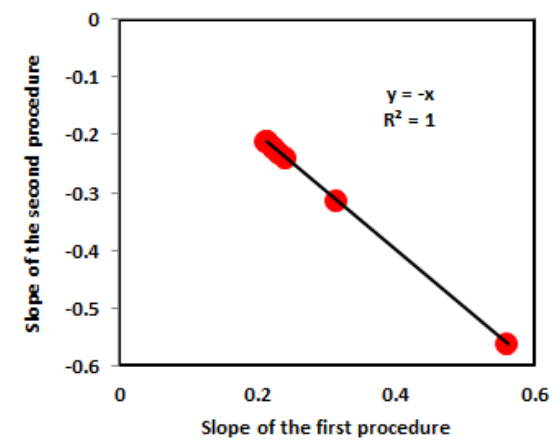

Figure12. Slope of the first procedure versus slope of the second procedure

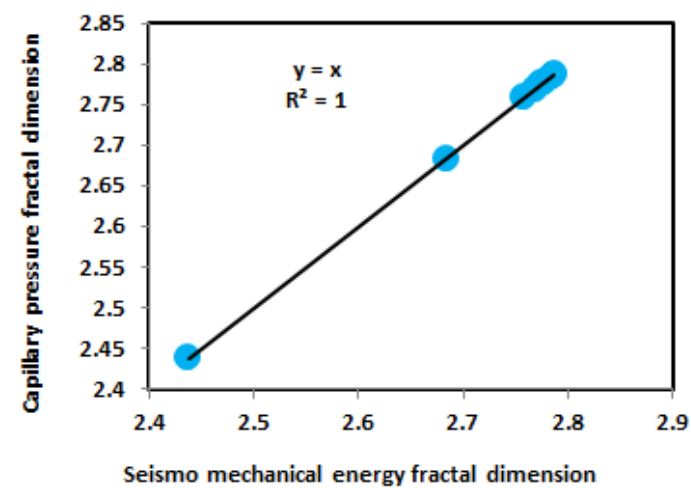

Figure13. Seismo mechanical energy fractal dimension versus capillary pressure fractal dimension 
Seismo Mechanical Energy Fractal Dimension for Characterizing Shajara Reservoirs of the PermoCarboniferous Shajara Formation, Saudi Arabia

Table1. Petrophysical model showing the three Shajara Reservoir Units with their corresponding values of seismo mechanical energy fractal dimension and capillary pressure fractal dimension

\begin{tabular}{|c|c|c|c|c|c|c|c|c|}
\hline Formation & Reservoir & Sample & $\begin{array}{l}\text { Porosity } \\
\%\end{array}$ & $\begin{array}{l}\mathrm{k} \\
\text { (md) }\end{array}$ & $\begin{array}{l}\text { Positive } \\
\text { slope of the } \\
\text { first } \\
\text { procedure } \\
\text { Slope=3-Df }\end{array}$ & $\begin{array}{l}\text { Negative } \\
\text { slope of the } \\
\text { second } \\
\text { procedure } \\
\text { Slope=Df-3 }\end{array}$ & $\begin{array}{l}\text { Seismo } \\
\text { mechanical } \\
\text { energy } \\
\text { fractal } \\
\text { dimension }\end{array}$ & $\begin{array}{l}\text { Capillary } \\
\text { pressure } \\
\text { fractal } \\
\text { dimension }\end{array}$ \\
\hline \multirow{10}{*}{ 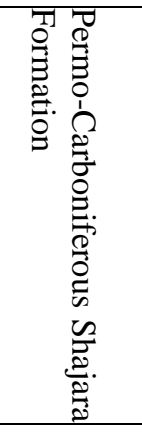 } & \multirow{3}{*}{$\begin{array}{l}\text { Upper } \\
\text { Shajara } \\
\text { Reservoir }\end{array}$} & SJ13 & 25 & 973 & 0.2128 & -0.2128 & 2.7872 & 2.7872 \\
\hline & & SJ12 & 28 & 1440 & 0.2141 & -0.2141 & 2.7859 & 2.7859 \\
\hline & & SJ11 & 36 & 1197 & 0.2414 & -0.2414 & 2.7586 & 2.7586 \\
\hline & \multirow{3}{*}{$\begin{array}{l}\text { Middle } \\
\text { Shajara } \\
\text { Reservoir }\end{array}$} & SJ9 & 31 & 1394 & 0.2214 & -0.2214 & 2.7786 & 2.7786 \\
\hline & & SJ8 & 32 & 1344 & 0.2248 & -0.2248 & 2.7752 & 2.7752 \\
\hline & & SJ7 & 35 & 1472 & 0.2317 & -0.2317 & 2.7683 & 2.7683 \\
\hline & \multirow{4}{*}{$\begin{array}{l}\text { Lower } \\
\text { Shajara } \\
\text { Reservoir }\end{array}$} & SJ4 & 30 & 176 & 0.3157 & -0.3157 & 2.6843 & 2.6843 \\
\hline & & $\mathrm{SJ} 3$ & 34 & 56 & 0.5621 & -0.5621 & 2.4379 & 2.4379 \\
\hline & & $\mathrm{SJ} 2$ & 35 & 1955 & 0.2252 & -0.2252 & 2.7748 & 2.7748 \\
\hline & & SJ1 & 29 & 1680 & 0.2141 & -0.2141 & 2.7859 & 2.7859 \\
\hline
\end{tabular}

\section{Conclusion}

The sandstones of the Shajara Reservoirs of the Permo-Carboniferous Shajara formation were divided here into three units based on seismo mechanical energy fractal dimension. The Units from base to top are: Lower Shajara Seismo Mechanical Energy Fractal Dimension Unit, Middle Shajara Seismo Mechanical Energy Fractal Dimension Unit, and Upper Shajara Seismo Mechanical Energy Fractal Dimension Unit. These units were also proved by capillary pressure fractal dimension. The fractal dimension was found to increase with increasing grain size and permeability owing to possibility of having interconnected channels.

\section{ACKNOWLEDGEMENT}

The author would to thank King Saud University, College of Engineering, Department of Petroleum and Natural Gas Engineering, Department of Chemical Engineering, Research Centre at College of Engineering, College of Science, Department of Geology, and King Abdullah Institute for research and Consulting Studies for their supports.

\section{REFERENCES}

[1] Frenkel J. On the theory of seismic and seismoelectric phenomena in a moist soil. Journal of physics, 1944, 3: 230-241. https://olemiss.edu/sciencenet/poronet/frenkel.pdf

[2] Li K, Williams W. Determination of pressure head function from resistivity data. Transport in Porous Media, 2007, 67: 1-15. https://link.springer.com/article/10.1007/s11242-006-0009-9

[3] Revil A, Jardani A. Seismo electric response of heavy oil reservoirs: theory and numerical modelling. Geophysical J International, 2010,180: 781-797.

https://academic.oup.com/gji/article/180/2/781/689125

[4] Dukhin A, Goetz P, Thommes M. Seismoelectric effect: a non-isochoric streaming current.1 Experiment. J Colloid Interface Sci, 2010,345: 547-553.

https://www.sciencedirect.com/science/article/pii/S0021979710001682

[5] Guan W, Hu H, Wang Z. Permeability inversion from low-frequency seismoelectric logs in fluid-saturated porous formations. Geophys Prospect,2012, 61: 120-133. https://onlinelibrary.wiley.com/doi/full/10.1111/j.1365-2478.2012.01053.x

[6] Hu H, Guan W, Zhao W. Theoretical studies of permeability inversion from seismoelectric logs. Geophysical Research Abstracts, 2012, 14: EGU2012-6725-1 2012 EGU General Assembly. https://ui.adsabs.harvard.edu/abs/2012EGUGA..14.6725H/abstract

[7] Borde C, S en echal P Barri ere J, Brito D, Normandin E et al., Impact of water saturation on seismoelectric transfer functions: a laboratory study of co-seismic phenomenon. Geophysical J International, 2015, 200: 1317-1335.

https://academic.oup.com/gji/article/200/3/1317/628077 
Seismo Mechanical Energy Fractal Dimension for Characterizing Shajara Reservoirs of the PermoCarboniferous Shajara Formation, Saudi Arabia

[8] Jardani A, Revil A. Seismoelectric couplings in a poroelastic material containing two immiscible fluid phases. Geophysical Journal International, 2015, 202: 850-870.

https://academic.oup.com/gji/article/202/2/850/587599

[9] Holzhauer J, Brito D, Bordes C, Brun Y, Guatarbes B. Experimental quantification of the seismoelectric transfer function and its dependence on conductivity and saturation in loose sand. Geophys Prospect, 2016, 65: 1097-1120

https://onlinelibrary.wiley.com/doi/full/10.1111/1365-2478.12448

[10] Rong Peng, Jian-Xing Wei, Bang-Rang Di, Pin-Bo Ding, ZiChun Liu. Experimental research on seismoelectric effects in sandstone. Applied Geophysics, 2016, 13: 425-436.

https://link.springer.com/content/pdf/10.1007\%2Fs11770-016-0570-0.pdf

[11] Djuraev U, Jufar S R, Vasant P (2017) Numerical Study of frequency-dependent seismo electric coupling in partiallysaturated porous media. MATEC Web of Conferences 87, 02001.

https://www.matec-

conferences.org/articles/matecconf/abs/2017/01/matecconf_encon2017_02001/matecconf_encon2017_020 01.html

[12] Alkhidir KEME. Seismo Magnetic Moment Fractal Dimension for Characterizing Shajara Reservoirs of the PermoCarboniferous Shajara Formation, Saudi Arabia World Scientific News, 2020, 139 (2):186-200. http://psjd.icm.edu.pl/psjd/element/bwmeta1.element.psjd-2d5681ab-6860-438b-a010-d28a0f013692

[13] Alkhidir KEME. Seismo Diffusion Coefficient Fractal Dimension for Characterizing Shajara Reservoirs of the Permo-Carboniferous Shajara Formation, Saudi Arabia. Research Journal of Nanoscience and Engineering, 2019, 3 (4):23-29.

https://www.sryahwapublications.com/research-journal-of-nanoscience-and-engineering/pdf/v3-i4/4.pdf

[14] Alkhidir KEME. Molar Enthalpy Fractal Dimension for Characterizing Shajara Reservoirs of the PermoCarboniferous Shajara Formation. Journal of Agriculture and Aquaculture. 2019, 1(1):1-8.

https://escientificpublishers.com/molar-enthalpy-fractal-dimension-for-characterizing-shajara-reservoirsof-the-permo-carboniferous-shajara-formation-JAA-01-0005

[15] Alkhidir KEME. Work Fractal Dimension for Characterizing Shajara Reservoirs of the PermoCarboniferous Shajara Formation, Saudi Arabia. 2019. Int J Environ \& Agri Sci 3:021.

https://bioaccent.org/environmental-agricultural-science/environmental-agricultural-science21.php

[16] Alkhidir KEME. Electro Kinetic Fractal Dimension for Characterizing Shajara Reservoirs of the Shajara Formation. Int J Nano Med \& Eng, 2018, 3(4):54-60.

http://biocoreopen.org/ijnme/Electro-kinetic-fractal-dimension-for-characterizing-Shajara-reservoirs-ofthe-Shajara-Formation.php

[17] Al-Khidir KE. On Similarity of Pressure Head and Bubble Pressure Fractal Dimensions for Characterizing Permo-Carboniferous Shajara Formation, Saudi Arabia. J Indust Pollut Toxic, 2018, 1(1): 102

http://www.annexpublishers.com/articles/JIPT/1102-On-Similarity-of-Pressure-Head-and-Bubble-

Pressure-Fractal-Dimensions-for-Characterizing-Permo-Carboniferous-Shajara-Formation-

Saudi\%20Arabia.pdf

Citation: Khalid Elyas Mohamed Elameen Alkhidir, (2020). "Seismo Mechanical Energy Fractal Dimension for Characterizing Shajara Reservoirs of the Permo-Carboniferous Shajara Formation, Saudi Arabia”, International Journal of Petroleum and Petrochemical Engineering (IJPPE), 6(1), pp.14-23, DOI: http://dx.doi.org/10.20431/2454-7980.0601002

Copyright: (C) 2020 Authors. This is an open-access article distributed under the terms of the Creative Commons Attribution License, which permits unrestricted use, distribution, and reproduction in any medium, provided the original author and source are credited 